\title{
Protective effects of polysialic acids (PSAs) and its mimics on the nervous system after injury
}

\author{
*1,2Wei-jiang Zhao, *2Jia-hui He, ${ }^{3}$ Shuang-xi Chen \\ WJ Zhao and JH He contributed equally to this work and are Co-First Authors \\ ${ }^{1}$ Wuxi School of Medicine, Jiangnan University, Wuxi, Jiangsu; ${ }^{2}$ Center for Neuroscience, Shantou \\ University Medical College, Shantou, Guangdong; ${ }^{3}$ Department of Neurology, The First Affiliated \\ Hospital, University of South China, Hengyang, Hunan, China
}

\begin{abstract}
Polysialic acid (PSA), a polymer of alpha-2,8 linked sialic acid residues, is a negatively charged macromolecular glycan mainly attached to neural cell adhesion molecules (NCAM). Studies have shown that PSA is not only essential for the development of normal brain circulation, but also for synaptic plasticity, learning and memory in adults. Although the occurrence, features, biosynthesis, and physiological roles of PSA and related effects on related diseases, including schizophrenia, bipolar disorder, neurodegenerative diseases and cancer, have been well reviewed, the important roles of PSA and its mimics in the regeneration of the nervous system following injury have not been well discussed. As a consequence, this article comprehensively reviews the effects of small organic compounds that simulate PSA, such as tegaserod and 5-nonyloxytryptamine (5-NOT), on the nervous system of mammals, suggesting that these mimetics may have tremendous therapeutic potential, especially for strategies aimed at tissue repair after injury of the nervous system.
\end{abstract}

Keywords: Polysialic acid; nervous system impairment; tegaserod; 5-NOT; neural regeneration

\section{INTRODUCTION}

Polysialic acid (PSA), a kind of unique carbohydrates, is a linear, homogenous poly alpha-2,8-linked sialic acid mainly attached to the neural cell adhesion molecules (NCAM) of the vertebrate nervous system through the typical $\mathrm{N}$-linked glycosides. ${ }^{1}$ In the developing and adult nervous system in higher vertebrates PSA can be expressed by migrating cells, such as olfactory interneuron precursors ${ }^{2}$, or dynamically expanding processes of Schwann cells or neurons in the synaptic plasticity region, and in stem cells in the subventricular zone..$^{3-5}$ PSA participates in learning, memory and synaptic plasticity, promotes cell motility and axon orientation and targeting, and mediates the interaction of NCAM with other molecules, such as brain-derived neurotrophic factors $(\mathrm{BDNF})^{6}, \alpha$-amino-3-hydroxy-5-methyl-4isoxazolepropionicacid (AMPA) receptors ${ }^{7}$, heparin sulfate proteoglycan (HSPG) ${ }^{8}$, histone $\mathrm{H} 1^{9}$, $\mathrm{N}$-methyl-D-aspartate (NMDA) receptor ${ }^{10}$, and myristoylated alanine-rich $C$ kinase(MARCKS) substrate. ${ }^{11}$ These functions of PSA are vital for the treatment of nervous disorders. Previous review first comprehensively outlines the occurrence, features, biosynthesis, and physiological roles of PSA, such as PSA formations of attractive filed for neurotrophic factors, growth factors, cytokines and proteins, and subsequently focuses on the related diseases, such as schizophrenia, bipolar disorder, neurodegenerative diseases and cancer. ${ }^{12}$ In the current review, we mainly concentrate on the effect of PSA and its mimics on the nervous system after injury.

When over-expressed in endogenous astrocytes and transplanted Schwann cells, virus-mediated PSA expression can enhance the formation of dendrites in Purkinje cells after injury and improve axonal regeneration after spinal cord injury (SCI). ${ }^{13}$ However, treatment with PSA transduction virus or application of PSA-expressing cells as therapeutic strategies has the disadvantages that PSA can be cleaved in vivo by neuraminidase and sialidase, such as sialidase NEU4 that is highly expressed in the central nervous system. ${ }^{14}$ In addition, it is sometimes difficult to isolate, purify, or produce

Address correspondence to: Wei-jiang Zhao, Wuxi School of Medicine, Jiangnan University, Wuxi, Jiangsu 212144, China. E-mail: weijiangzhao @ jiangnan. edu.cn; Shuang-xi Chen, Department of Neurology, The First Affiliated Hospital, University of South China, Hengyang, Hunan 421001, China. E-mail: dnafenzi@163.com

Date of Submission: 7 February 2021; Date of Acceptance: 2 May 2021

https://doi.org/10.54029/2021znh 
PSA from biological sources, especially when a defined PSA with a certain number of sialic acid residues is needed..$^{15}$ Therefore, compounds that structurally and functionally mimic PSA from small organic molecular libraries play a key role in promoting functional recovery and plasticity after peripheral and central nervous system injury.

\section{THEDEFINATIONAND BASIC STRUCTURE OF POLYSIALIC ACID}

Sialic acid, a derivative of neurominic acid, is an acidic amino sugar containing 9 carbon atoms and a pyranose structure. ${ }^{16}$ The PSA is a linear homopolymer of sialic acid monomers linked by $\alpha-2,8$ ketosidic linkages. ${ }^{17}$ The two monomers of the PSA terminal, which are next to each other and connected by the alpha ketone bond, form the lactone ${ }^{18}$ and play a certain role in the stability of PSA. The biochemical properties of PSA such as carrying of negative charges, space occupancy, and carrying of water and other ions, mainly depend on the carboxyl groups of sialic acid. Studies have shown that PSA is involved in cell adhesion, migration, neurite outgrowth, nerve bifurcation, neuronal orientation, synapse formation, neuritogenesis and other functions. ${ }^{19,20}$ The migration promoting effect of PSA is more common at the developmental stages of brain. For example, the migration of the luteinizing hormone releasing hormone $(\mathrm{GnRH})$ cells to the forebrain depends on polysialic acid. ${ }^{21}$ (Figure 1)

\section{MOLECULES INTERACTING WITH POLYSIALIC ACID AND THE SIGNALING PATHWAY}

PSA can regulate the activity of many cell adhesion molecules such as integrins, cadherins, and other immunoglobulin superfamily molecules involved in neuronal cell adhesion ${ }^{21}$, while it mainly attaches to NCAM. ${ }^{24} \mathrm{NCAM}$ is a cell surface glycoprotein composed of five immunoglobulin (Ig)-like domains and two fibronectin III repeat units. PSA is linked to NCAM by glycosides on the N-glycosylation site on Ig-5. ${ }^{21}$ PSA can form a larger hydration radius as it is hydrophilic and carries many negative charges..$^{25}$ The presence of PSA increases the distance between cell membranes during NCAM-NCAM interaction, causing other adhesion molecules on the membrane to lose adhesion ability due to

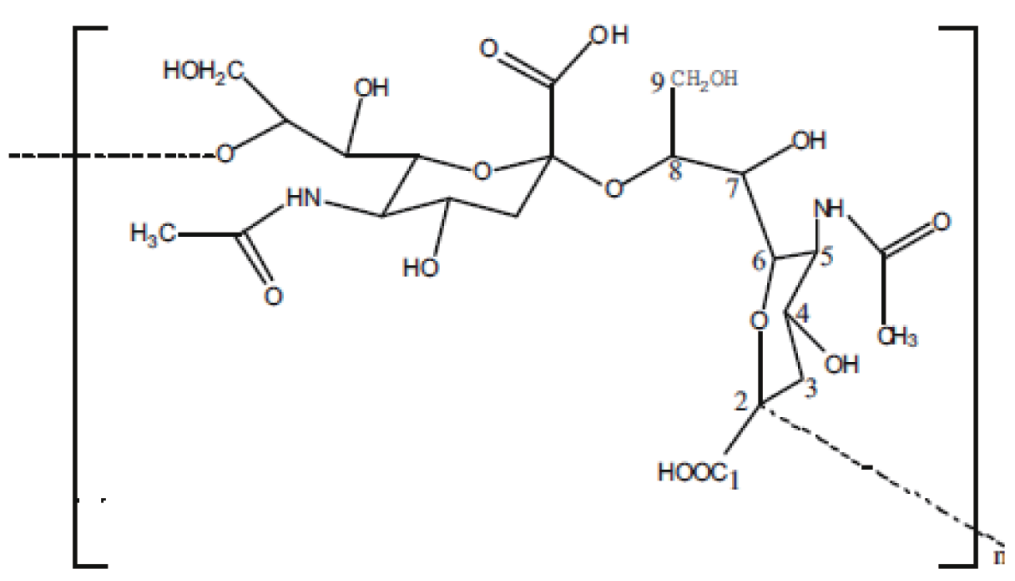

$\alpha-2,8$-linked PSA

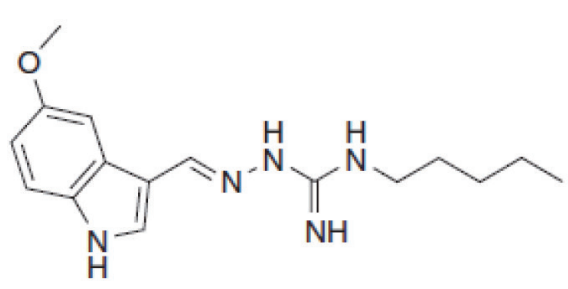

Tegaserod

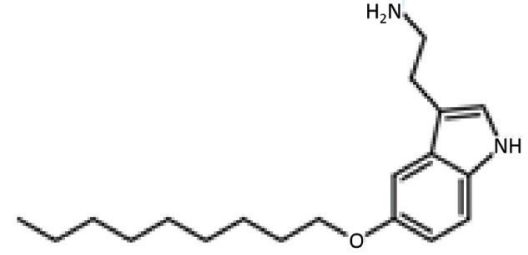

5-nonyloxytryptamine

Figure 1. Structures of $\alpha$-2,8-linked PSA, tegaserod and 5-nonyloxytryptamine[22, 23]. 
excessive distance, thus reducing the adhesion between cell membranes. However, deletion of PSA by specific exonuclease EdnoN can enhance the adhesion between membranes. ${ }^{26}$ Therefore, the presence or absence of PSA can directly or indirectly regulate the adhesion between cell membranes. Since PSA is expressed only in the postembryonic and early postnatal stages, it plays an important role in regulating the development of the nervous system. ${ }^{27}$ (Figure 2).

\section{REPARING EFFECT OF POLYSIALIC ACID MIMICS ON NERVOUS SYSTEM INJURY}

PSA mimetic peptides have been well developed by performing phage display screening ${ }^{28}$, and may have therapeutic effect. (Figure 3) The peptides and small compound mimics of PSA have a multiple of advantages in drug development for therapies in the treatment of human diseases. Purified synthetic compounds are non-xenogeneic as compared to proteins or molecules purified from animals and bacteria or recombinantly generated in E.coli, insect or mammalian cells. Application of PSA mimetic peptides discovered by screening of phage display libraries with PSA monoclonal antibodies ${ }^{28,29}$ or small molecules can promote functional recovery and plasticity after SCI and femoral nerve injury. ${ }^{2,24,29-32}$

Tegaserod, a FDA-approved drug for treatment of irritable bowel syndrome and constipation ${ }^{33}$ by stimulating the $5-\mathrm{HT}_{4}$ receptor on enteric neurons ${ }^{34,35}$, has been screened as a specific PSA mimicking small organic compound from a library of small organic molecules. ${ }^{28}$ Two different functions of tegaserod can be functionally distinguished from each other. ${ }^{24}$ PSA mimicking activity of tegaserod was confirmed in the primary culture of neurons and Schwann cells from both central and peripheral nervous systems of mice, and this effect was independent of its original function as the 5-serotonin (5-HT) receptor agonist. ${ }^{32}$ Also, tegaserod can enhance locomotor recovery and regrowth sprouting of axons when applied at the lesion site of mice with a femoral nerve injury of the peripheral nervous system in vivo. ${ }^{32}$ In a mouse model of severe compression-induced SCI, the regenerative function of the mice treated with tegaserod was significantly enhanced when compared to the control-treated mice. ${ }^{24}$ These experiments validate the potential of tegaserod in treating nervous system injuries in mammals.

Studies have reported that PSA cyclic mimetic peptide (PR-21) can display PSA-like biological functions, including neurite outgrowth, fasciculation and guidance, and increased the migration of transplanted neural progenitors along the rostral migratory stream. ${ }^{28}$ After dorsal hemisection at the T9 level of the adult mouse, delivery of PR-21 to the lesion site decreased the recovery time, enhanced motor functions, sensorimotor control, and coordination of the hindlimb with the forelimb when compared to the control peptide-treated mice. ${ }^{31}$ At the cellular level, PR-21 increases the serotonergic

polysialic acid (PSA)

$\downarrow$ alter

the adhesion of nerve cell adhesion molecules

(NCAM) in the nervous system

involved in

cell adhesion, migration, neurite outgrowth, neuronal orientation, synapse formation

$\downarrow$ regulate

the development

and remodeling of

the nervous system

Figure 2. Physiological pattern of PSA. 


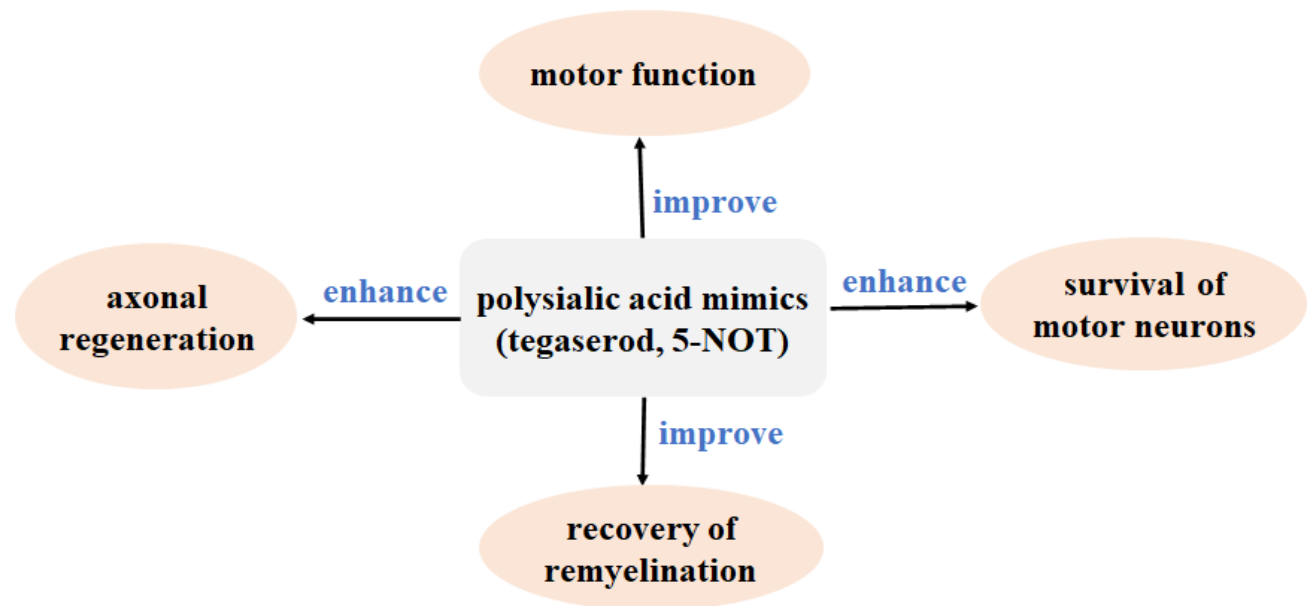

Figure 3. Schematic diagram demonstrating the therapeutic effect of PSAs mimics.

axon density at and caudal to the lesion site and reduces reactive gliosis in vivo. In an in vitro model of reactive astrocytes, PR-21 increased NCAM expression in enhanced glial fibrillary acidic protein (GFAP)-positive cells. ${ }^{30}$

The 5-nonyloxytryptamine (5-NOT), which was identified using a small organic molecule library, plays a similar role to that of colominic acid in the aspect of stimulating regeneration after SCI. ${ }^{7}$ In addition, it is as effective as the PSA cyclic mimetic peptide (PR-21) in enhancing regeneration after $\mathrm{SCI}^{30,31}$, and it can be applied at lower concentration and cannot be degraded by peptidases. In a mouse SCI model, the 5-NOT can yield an overall functional recovery of approximately $80 \% .^{2}$ Based on the above findings, tegaserod and 5-NOT are expected to become candidate drugs for treatment of the nervous system injury (Figure 2).

\section{CONCLUSION}

PSA is not only an important glycan in the nervous system development and synaptic plasticity, but also in regeneration after injury. Studies indicate that PSA mimicking small organic compounds, such as tegaserod and 5-NOT, are potential drugs in the development of novel neurological therapies. Their application creates favorable conditions for axonal regeneration and remyelination to promote functional recovery after injury. Therefore, such compounds may be applied as a combined therapeutic strategy involved in the recovery promotion of acute nervous system injury and repair of neurodegenerative diseases, laying the foundation for PSA and the mimetic peptides as novel candidates for the treatment of the diseases related to neuronal injury in the future.

\section{DISCLOSURE}

Financial support: The authors are grateful to the National Natural Science Foundation of China (Grant Nos. 81471279 and 81171138 to W-JZ), The Research Start-Up Fund of Wuxi School of Medicine, Jiangnan University (grant no. 1286010242190060 to W-JZ), and the Scientific Research Fund Project of Hunan Provincial Health Commission (Grant No. 20201963 to S-XC) for support.

Conflicts of interest: None

\section{REFERENCES}

1. Nakata D, Troy FA, 2nd. Degree of polymerization (DP) of polysialic acid (polySia) on neural cell adhesion molecules (N-CAMS): development and application of a new strategy to accurately determine the DP of polySia chains on N-CAMS.J Biol Chem 2005; 280: 38305-16.

2. Saini V, Lutz D, Kataria H, Kaur G, Schachner M, Loers G. The polysialic acid mimetics 5-nonyloxytryptamine and vinorelbine facilitate nervous system repair. Sci Rep 2016; 6: 26927.

3. Roche PH, Figarella-Branger D, Daniel L, Bianco N, Pellet W, Pellissier JF. Expression of cell adhesion molecules in normal nerves, chronic axonal neuropathies and Schwann cell tumors. J Neurol Sci 1997; 151: 127-33.

4. Durbec P, Cremer H. Revisiting the function of PSANCAM in the nervous system. Mol Neurobiol. 2001; 24: 53-64.

5. Angata K, Fukuda M. Roles of polysialic acid in migration and differentiation of neural stem cells. Methods Enzymol 2010; 479: 25-36.

6. Muller D, Djebbara-Hannas Z, Jourdain P, et al. Brain-derived neurotrophic factor restores long-term potentiation in polysialic acid-neural cell adhesion 
molecule-deficient hippocampus. Proc Natl Acad Sci U S A 2000; 97: 4315-20.

7. Vaithianathan T, Matthias K, Bahr B, Schachner M, Suppiramaniam V, Dityatev A, Steinhaüser C. Neural cell adhesion molecule-associated polysialic acid potentiates alpha-amino-3-hydroxy5-methylisoxazole-4-propionic acid receptor currents. J Biol Chem 2004; 279: 47975-84.

8. Storms SD, Rutishauser U. A role for polysialic acid in neural cell adhesion molecule heterophilic binding to proteoglycans. J Biol Chem 1998; 273: 27124-9.

9. Mishra B, von der Ohe M, Schulze C, et al. Functional role of the interaction between polysialic acid and extracellular histone H1. J Neurosci 2010; 30: 1240013.

10. Hammond MS, Sims C, Parameshwaran K, Suppiramaniam V, Schachner M, Dityatev A. Neural cell adhesion molecule-associated polysialic acid inhibits NR2B-containing N-methyl-D-aspartate receptors and prevents glutamate-induced cell death. J Biol Chem 2006; 281: 34859-69.

11. Theis T, Mishra B, von der Ohe M, et al. Functional role of the interaction between polysialic acid and myristoylated alanine-rich $C$ kinase substrate at the plasma membrane. J Biol Chem 2013; 288: 6726-42.

12. Sato C, Kitajima K. Polysialylation and disease. $\mathrm{Mol}$ Aspects Med 2020: 100892.

13. Zhang Y, Ghadiri-Sani M, Zhang X, Richardson PM, Yeh J, Bo X. Induced expression of polysialic acid in the spinal cord promotes regeneration of sensory axons. Mol Cell Neurosci 2007; 35: 109-19.

14. Takahashi K, Mitoma J, Hosono M, et al. Sialidase NEU4 hydrolyzes polysialic acids of neural cell adhesion molecules and negatively regulates neurite formation by hippocampal neurons. J Biol Chem 2012; 287: 14816-26.

15. Loers G, Astafiev S, Hapiak Y, et al. The polysialic acid mimetics idarubicin and irinotecan stimulate neuronal survival and neurite outgrowth and signal via protein kinase C. J Neurochem 2017; 142: 392406.

16. Iwasaki M,Inoue S,Troy FA.Anew sialic acid analogue, 9-O-acetyl-deaminated neuraminic acid, and alpha -2,8-linked O-acetylated poly(N-glycolylneuraminyl) chains in a novel polysialoglycoprotein from salmon eggs. J Biol Chem 1990; 265: 2596-602.

17. McGuire EJ, Binkley SB. The structure and chemistry of colominic acid. Biochemistry 1964; 3: 247-51.

18. Manzi AE, Higa HH, Diaz S, Varki A. Intramolecular self-cleavage of polysialic acid. J Biol Chem 1994; 269: 23617-24.

19. Rutishauser U, Landmesser L. Polysialic acid in the vertebrate nervous system: a promoter of plasticity in cell-cell interactions. Trends Neurosci 1996; 19: 422-7.

20. Chen SX, He JH, Mi YJ, Shen HF, Schachner M, Zhao WJ. A mimetic peptide of $\alpha 2,6$-sialyllactose promotes neuritogenesis. Neural Regen Res 2020; 15: 1058-65.

21. Wu JR, Zhan XB, Zheng ZY, Jia W. Recent progress in polysialic acid and sialic acid research. Sheng $W u$ Jia Gong Guo Cheng 2007; 01: 20-6.

22. Wu J, Zhan X, Liu L, Xia X. Bioproduction, purification, and application of polysialic acid. Appl Microbiol Biotechnol 2018; 102: 9403-9.

23. Tack J, Camilleri M, Chang L, et al. Systematic review: cardiovascular safety profile of 5-HT(4) agonists developed for gastrointestinal disorders. Aliment Pharmacol Ther 2012; 35: 745-67.

24. Pan HC, Shen YQ, Loers G, Jakovcevski I, Schachner M. Tegaserod, a small compound mimetic of polysialic acid, promotes functional recovery after spinal cord injury in mice. Neuroscience 2014; 277 : 356-66.

25. Cunningham BA, Hoffman S, Rutishauser U, Hemperly JJ, Edelman GM. Molecular topography of the neural cell adhesion molecule N-CAM: surface orientation and location of sialic acid-rich and binding regions. Proc Natl Acad Sci U S A 1983; 80: 3116-20.

26. Yang $P$, Yin $X$, Rutishauser U. Intercellular space is affected by the polysialic acid content of NCAM. $J$ Cell Biol 1992; 116: 1487-96.

27. Zhou WB, Zhou CF. Role of neural cell adhesion molecule and polysialic acid on the neuronal development and regeneration. Sheng Li Ke Xие Jin Zhan 1996; 27: 118-22.

28. Torregrossa P, Buhl L, Bancila M, et al. Selection of poly-alpha 2,8-sialic acid mimotopes from a random phage peptide library and analysis of their bioactivity. J Biol Chem 2004; 279: 30707-14.

29. Mehanna A, Mishra B, Kurschat N, et al. Polysialic acid glycomimetics promote myelination and functional recovery after peripheral nerve injury in mice. Brain 2009; 132: 1449-62.

30. Marino P, Norreel JC, Schachner M, Rougon G, Amoureux MC. A polysialic acid mimetic peptide promotes functional recovery in a mouse model of spinal cord injury. Exp Neurol 2009; 219: 163-74.

31. Mehanna A, Jakovcevski I, Acar A, et al. Polysialic acid glycomimetic promotes functional recovery and plasticity after spinal cord injury in mice. Mol Ther 2010; 18: 34-43.

32. Bushman J, Mishra B, Ezra M, et al. Tegaserod mimics the neurostimulatory glycan polysialic acid and promotes nervous system repair. Neuropharmacology 2014; 79: 456-66.

33. Müller-Lissner SA, Fumagalli I, Bardhan KD, et al. Tegaserod, a 5-HT(4) receptor partial agonist, relieves symptoms in irritable bowel syndrome patients with abdominal pain, bloating and constipation. Aliment Pharmacol Ther 2001; 15: 1655-66.

34. Liu M, Geddis MS, Wen Y, Setlik W, Gershon MD. Expression and function of 5-HT4 receptors in the mouse enteric nervous system. Am J Physiol Gastrointest Liver Physiol 2005; 289: G1148-63.

35. Liu MT, Kuan YH, Wang J, Hen R, Gershon MD. 5-HT4 receptor-mediated neuroprotection and neurogenesis in the enteric nervous system of adult mice. J Neurosci 2009; 29: 9683-99. 